\title{
Análisis de riesgo a la salud de una instalación petrolera del Suroeste de México
}

\author{
R. Iturbe-Argüelles, R.Ma. Flores-Serrano y L.G. Torres-Bustillos \\ Instituto de Ingeniería, UNAM. Coordinación de Ingeniería A mbiental. Grupo Saneamiento de Suelos y Acuíferos. \\ E-mail: ria@ pumas.iingen.unam.mx
}

(recibido: abril de 2005; aceptado: septiembre de 2005)

\section{Resumen}

En la Terminal de Almacenamiento y Distribución de combus ti bles, (TAD), tales como el diesel y la gasolina, localizada al Suroeste de México, se detectó contaminación por hidrocarburos a raíz de una auditoria ambiental, por lo que se solicitó un estudio completo de la contaminación en la TAD. A partir de los resultados de este trabajo, se puede decir que el suelo de la TAD se encuentra contaminado por hidrocarburos totales de petróleo (HTP) en concentraciones de ND a 59,000 mg/kg. No se detectó la presencia de benceno, tolueno, etilbenceno y xileno (BTEX), metil terbutil éter (MTBE) ni metil-teramil éter (MTE). De los 16 hidrocarburos aromáticos policíclicos (HAP) analizados, sólo se encontró la presencia de naftaleno en un punto, a una concentración por debajo de $1 \mathrm{mg} / \mathrm{kg}$. Se identificó la presencia de $\mathrm{Fe}, \mathrm{Pb}$ y $\mathrm{Zn}$ en el suelo, pero los índices de geo-acumulación calculados sugieren que se trata de concentraciones naturales del suelo y no de contaminación alguna. Se encontraron HTP en las aguas subterráneas, solo en uno de los 6 pozos por arriba del nivel recomendado de $10 \mathrm{mg} / \mathrm{l}$. También se encontró que el Fe excede el valor sugerido de $0.3 \mathrm{mg} / \mathrm{l}$ en 5 de los 6 pozos estudiados. Se encontró que el pH de las aguas subterráneas no está relacionado con el nivel de metales encontrados (Fe y Zn), pero sí con el contenido de HTP. El análisis de riesgo a la salud indicó que las concentraciones de naftaleno y zinc, presentes en el suelo y agua subterránea, no representan un riesgo para la salud de los habitantes de las colonias aledañas ni para los trabajadores de la TAD. Sin embargo, dados los altos niveles de concentración de HTP al norte de la TAD, se recomendó el saneamiento de un volumen de aproximadamente $8,000 \mathrm{~m}^{3}$ mediante el proceso de biopilas.

Descriptores: Agua subterránea, contaminación de suelos, hidrocarburos, riesgo a la salud, técnicas de saneamiento de suelos.

\footnotetext{
Abstract

Dur ing an $E n$ vi ron men tal $A$ u dit in an oil stor ageand dis tri bu tion sta tion (TAD ) lo cated at South-west M ex ico, hy dro car bon con tam i na tion was detected. Because of it, a com pletestu dy of thesite's con tami na tion was required. From this work's results, it can bestated that TA D sub soil is con tam i nated by to tal petro leum hy dro car bons (TPH ), in con cen tra tion from N D to 59,000 $\mathrm{mg} / \mathrm{kg}$. N ei ther ben zene, to lu ene, ethylbenzeneor xylenes (BTEX), nor methyl tert-butyl ether (M TBE), nor methyl tert-amyl ether (M TE) werede tected. From the 16 poly cyclic ar o matic hy drocar bons (PAH) an a lyzed, only naph tha lenewas found in onesam plepoint at a con cen tra tion be low $1 \mathrm{mg} / \mathrm{kg}$. The pres ence of $\mathrm{Fe}, \mathrm{Pb}$, and $\mathrm{Zn}$ was iden ti fied in the sub soil, but the cal cu lated geo- accumulation in dexes sug gest a nat $u$ ral con cen tra tions and not a con tam i na tion's product. TPH were found in groundwaters, but only in one of the six sam pled wells over the rec om mended level of $10 \mathrm{mg} / \mathrm{l}$. It was also found that $F$ e con cen tra tions ex ceed the sug gested value of $0.3 \mathrm{mg} / \mathrm{l}$ in five of the six sam pled wells. It was de ter mined that ground wa ter's $\mathrm{pH}$ value is not linked to the
} 
met als con cen tra tion level, but with theTPH con cen tra tion. $H$ ealth risk assess ment in dic ated that naph tha lene, and zinccon cen tra tions, pres ent in wa ter and sub soil, do not rep resent a health risk nei ther for theneigh bour hood in hab it ants, nor for theTAD work ers. N ever theless, given the high TPH con cen tra tions in theTAD ' s north side, the remediation of $8,000 \mathrm{~m}^{3}$ of sub soil by means of a biopiles sys tem was rec om mended.

Keywords: G round wa ter, health risk, hy drocar bons, soil con tam ina tion, soil remediation tech niques.

\section{Introducción}

\section{Contaminación de suelos con hidrocarburos de petróleo}

La operación de sitios de exploración, producción, refinación y almacenamiento de crudo, frecuentemente trae como consecuencia derrames de hidrocarburos que tienen como destino final suelos y cuerpos de agua. Para el año 2001, PEMEX reportó la cantidad de 1,500 derrames de hidrocarburos, por un total de aproximadamente 6,250 toneladas de crudo (PEMEX 2001). Estas cifras abarcan solamente las operaciones de exploración, producción y refinación. Las estaciones de almacenamiento y distribución, también están sujetas a derrames de crudo y combustibles, aunque no se tengan reportes suficientes al respecto.

En el ámbito internacional, se han reportado trabajos relacionados con la caracterización de suelo y aguas subterráneas en sitios relacionados con la industria del petróleo, así como estudios de riesgo a la salud de los mismos. Adenyi y Afolabi (2002), reportaron las concentraciones de hidrocarburos y metales pesados en áreas de gasolineras, talleres mecánicos y sitios de almacenamiento de productos derivados del petróleo, en comparación con los contenidos de dos suelos de referencia no expuestos a la contaminación. Por otro lado, Ekundayo y Obuekwe (1997), caracterizaron una zona de Ni ge ria, donde derrames continuos de hidrocarburos han contaminado el suelo de la región. Shriadah (1999), describió la contaminación de los suelos de una zona de los Emiratos Unidos Árabes, debido al paso de autotanques que transportan crudo en la región. En los Estados Unidos, Sharma et al. (2002), han reportado la contaminación de la Costa del Sur de Texas por derrames de hidrocarburos. Bakker et al. (2000), identificaron y caracterizaron hidrocarburos aromáticos policílicos en suelos cercanos a una refinería de petróleo en Zelzate, Bélgica. Chen et al. (2000), reportaron un modelo multifase y multicomponentes que permite el estudio de riesgo en zonas contaminadas con petróleo y sus derivados.

En México, Gutiérrez y Zavala (2001) y Adams et al. (1999), entre otros, han caracterizado suelos de zonas petroleras de Tabasco, cubriendo aspectos como los rasgos hidromórficos de los suelos contaminados y el potencial de biorremediación que tienen dichos suelos. Además de estos autores, Lesser y Saval (2000), han reportado la caracterización de la contaminación con hidrocarburos en suelos arcillosos en una estación de almacenamiento y distribución de combustibles de México. Por otra parte, Iturbe y colaboradores reportaron estudios de caracterización de las terminales de almacenamiento y distribución de Puebla, Puebla (Iturbe et al, 1999), Zacatecas, Zacatecas (Iturbe et al, 2003a) y Morelia, Michoacán (Iturbe et al, 2003b).

La Terminal de Almacenamiento y Distribución (TAD) está localizada al Suroeste de México. Después de una auditoría ambiental llevada a cabo en la antigua TAD, surgió la necesidad de llevar a cabo un estudio orientado a la caracterización del suelo y agua subterránea en el área de la instalación. Una de las principales deficiencias establecidas en la auditoría ambiental se refiere a la presencia de hidrocarburos al poniente de la instalación, debida a un derrame de combustibleen el pasado. También se estableció la presencia de hidrocarburos cerca de la fosa de aceites, al Noreste de la TAD. De esta forma, los objetivos principales de este trabajo son:

a) Evaluar los niveles de contaminación del suelo y agua subterránea de la antigua TAD, 
b) Elaborar un estudio de riesgo a la salud y establecer los niveles de limpieza requeridos, así como

c) Proponer opciones de saneamiento.

\section{Caracterización del sitio}

La TAD se ubica al Suroeste de México. El clima de la zona corresponde a un clima cálido y sub-húmedo. Debido a sus condiciones de temperatura se de fine como cálido, ya que presenta una temperatura media anual sobre los $22^{\circ} \mathrm{C}$ y la temperatura media del mes más frío está sobre $18^{\circ} \mathrm{C}$. La precipitación pluvial promedio de los últimos 20 años es de 1,403 mm. La humedad relativa varía entre el 76 y $80 \%$. El municipio pertenece a la región hidrológica $\mathrm{RH}-19$ que forma parte de las cuencas del río Atoyac. El coeficiente de escurrimiento de la zona es de $10 \%$.

El material granular que corresponde a esta región es de arena con grano grueso, medianamente consolidado, con alta permeabilidad. El material consolidado con bajas posibilidades de funcionar como acuífero, está formado por rocas ígneas, sedimentarias y metamórficas. Las rocas sedimentarias tales como lutitas, calizas y areniscas se clasificaron como de baja permeabilidad por el contenido de material arcilloso y escaso fracturamiento.

Las rocas metamórficas por su génesis y fracturamiento poco profundo y escaso se consideran como malas conductoras y almacenadoras de agua. La corriente subterránea presenta una dirección hacia el Océano Pacífico. El nivel freático de la zona de la ter mi nal se encuentra entre los 4 y $6 \mathrm{~m}$ de profundidad. Es importante mencionar que la terminal está construida junto a un cerro. Los tanques de almacenamiento se encuentran instalados en la parte más alta, mientras que la zona contaminada es la más baja del sitio.

\section{Materiales y métodos}

$$
\text { M uestreo de agua y suelo }
$$

Las muestras de suelo se tomaron en 22 puntos distribuidos en la TAD, incluyendo estaciona- mientos, área de tanques de almacenamiento, zona de llenaderas y oficinas varias. Las muestras fueron tomadas entre la superficie y $3.5 \mathrm{~m}$ de profundidad. Se empleó un equipo de perforación neumático. El equipo cuenta con un motor de 12 HP y velocidad vari able con una presión de trabajo de 2,000 libras por pulgada cuadrada. Los parámet ros evaluados en las muestras de suelos fueron hidrocarburos totales del petróleo, perfil de hidrocarburos que incluye las fracciones de diesel, gasolina, MTBE y MTE y a los BTEX. Adicionalmente, se analizaron los 16 hidrocarburos aromáticos policíclicos no rmados por USEPA: acenafteno, fenantreno, fluoranteno, fluoreno, antraceno, indeno $(1,2,3 c, d)$ pireno, acenaftileno, naftaleno, pireno, benzo (a) antraceno, benzo (a) pireno, benzo (a) fluoranteno, benzo $(\mathrm{g}, \mathrm{h}, \mathrm{i})$ perileno, benzo $(\mathrm{k})$ fluoranteno, criseno y dibenzo $(a, h)$ antraceno. En las mismas muestras también se analizaron cuatro metales pesados: $\mathrm{Fe}, \mathrm{Cr}, \mathrm{Pb}$ y $\mathrm{Zn}$. El agua se muestreó en 6 pozos de monitoreo $(20 \mathrm{~m}$ de profundidad) ya existentes. Los parámetros analizados en el agua fueron $\mathrm{pH}, \mathrm{HTP}, \mathrm{Pb}, \mathrm{Fe}, \mathrm{Cr} \mathrm{Zn}, \mathrm{y}$ explosividad de los vapores. La explosividad de las muestras en ambas matrices fue medida con un equipo COSMOS XP-311 ALPHA A (New Cosmos Elec tric Co. Ltd, Japón).

\section{Técnicas analíticas}

Las muestras de suelo fueron caracterizadas de acuerdo a las técnicas analíticas propuestas por USEPA: EPA418.1 para los TPHs, EPA6010 para los metales pesados, y la EPA8240 para las fracciones de hidrocarburos. Los HAP s fueron analizados con el método EPA8100. Para las aguas se emplearon los siguientes métodos: EPA6010 para metales pesados y EPA418.1 para los HTP.

\section{Análisis de riesgo a la salud}

Para conocer el nivel de riesgo de la población en contacto con el suelo y el agua subterránea de las zonas aledañas a la TAD, así como de los trabajadores de la misma, a través de diferentes vías de exposición (contacto dérmico, inhalación de vapores 0 polvos, ingestión de suelo 0 agua subterránea contaminados por hidrocarburos), se requiere aplicar una evaluación de riesgo a la salud. La evaluación o análisis de riesgo a la salud 
se define como la caracterización de los efectos potenciales adversos a la salud humana, debidos a las exposiciones humanas a peligros ambientales (NRC, 1983). Esta caracterización se tra duce en un estimado numérico de riesgo (Crane y Tuchman, 1993).

El producto final de una evaluación de este tipo, es un número adimensional que indica la probabilidad de que un individuo desarrolle cáncer o sufra efectos no carcinógenos durante su vida media. Para compuestos carcinógenos se ha establecido como riesgo aceptable un valor de $1 \mathrm{x}$ $10^{-6}$ (probabilidad de que un individuo en un millón desarrolle cáncer).

El análisis de riesgo a la salud (ARS) fue llevado a cabo con ayuda del programa comercial RBCA Tool Kit for ChemicalReleases (GSI, 1998). No se incluyeron las concentraciones de HTP ni las mezclas de hidrocarburos (diesel, gasolina, etc.), ya que sólo los compuestos individuales pueden ser tomados en cuenta. Se definieron dos tipos de receptores: receptores on-site (aquellos que se encuentran dentro de la fuente de contaminación) y receptores off-site (aquellos que se encuentran fuera de la fuente de contaminación).

Los receptores on-site son los trabajadores de la TAD y los off-site son la gente que vive en los alrededores. Los receptores on-site se evaluaron con uso comercial de suelos, considerando también las actividades de construcción. Los trabajadores off-site, fueron evaluados como usuarios de suelo residencial.

De acuerdo con la ASTM (1996), el riesgo individual considerado como aceptable para cada compuesto químico dentro del área de estudio fue $1 \mathrm{x}$ 10-6 para las clases A y $B$ de los compuestos carcinogénicos y de $1 \times 10^{5}$ para los carcinogénicos clase $C$. El riesgo total acumulado considerado como aceptable es $1 \times 10^{-5}$. Para los compuestos no carcinogénicos (clases $D$ y $E$ ), al cociente de peligrosidad (HQ) y al índice de peligrosidad (HI) se les asignó un valor de 1.0. Cuando se aplicó el modelo, no se consideró degradación, ya que los receptores off-site se encuentran tan cerca de la fuente de contaminación que era necesario hacer más restrictivo el cálculo.

\section{Resultados y discusión}

Suelo

Identificación de posibles fuentes emisoras de hidrocarburos

En la zona Noroeste, al levantar la capa su per fi cial de suelo se observó presencia de contaminación. Se encontró un fuerte olor a gasolina. En la fosa de separación, el agua presenta una gruesa capa de hidrocarburos (puntos 1, 2, 3 y 4). Al lado del almacén hay una zanja de aproximadamente $0.6 \mathrm{~m}$ de profundidad, en donde se aprecia un horizonte contaminado con hidrocarburos de aproximadamente $0.3 \mathrm{~m}$ de la superficie del suelo con $0.05 \mathrm{~m}$ de espesor.

En la zona Norte se observaron pequeñas fugas en varias mangueras. Al perforar para obtener las muestras de suelo se percibió un fuerte olor a hidrocarburo (puntos 14, 15, 16 y 17). En la zona Noreste se ubica la fosa de decantación con agua de lluvia en su in te rior. La fosa está recubierta pero se observan algunas fracturas. Al costado Oriente se encuentra un almacenamiento de chatarra colocado sobre concreto y terreno natural y al costado Poniente se encuentra un almacén de llantas usadas (puntos 9, 10 y 11).

En la zona Oriente se encuentra el área de tanques de almacenamiento. En general, es una zona que se aprecia en buen estado, sin manchas superficiales. Con respecto a las zonas Sur y Suroeste, durante el muestreo de suelo se encontraban en remodelación. Se observaron algunas manchas superficiales en la zona, al Norte del estacionamiento de pipas (puntos de muestreo 18, 19,20 y 21).

\section{Distribución de las fracciones orgánicas (HTP, diesel, gasolina e HAP) y niveles de explosividad}

En la tabla 1 se resumen los valores de HTP, die sel, gasolina e HAP (naftaleno) encontrados en el suelo de la TAD. El resto de los compuestos orgánicos evaluados, no estuvieron presentes en los suelos. Como se puede observar, los HTP se encontraron en concentraciones de ND a 59,213 mg/kg, con un 
promedio de 3,954+/-13,241 mg/kg. La mediana de estos valores fue $14.5 \mathrm{mg} / \mathrm{kg}$. En la figura 1 se muestra la distribución espacial de estos valores. Aquí, el punto de mayor concentración de HTP fue el punto 1 localizado a un costado de las oficinas de la antigua TAD, donde se reportó un derrame accidental de combustible en años pasados. La concentración de HTP va disminuyendo gradualmente, pero cubre las áreas aledañas al laboratorio, estacionamiento exterior, estacionamiento de pipas y una fracción de la zona de llenaderas. Otras áreas con una importante concentración de HTP son los puntos 2, 4, 15 y 16. En el resto de las instalaciones, la concentración es menor a los $150 \mathrm{mg} / \mathrm{kg}$. Solamente se detectaron fracciones específicas de los HTP en algunos puntos. En los puntos 2,4 y 15 se detectó die sel en concentraciones de hasta $5,272 \mathrm{mg} / \mathrm{kg}$. En promedio, se detectó $437+/-1,277 \mathrm{mg} / \mathrm{kg}$ de este contaminante. Sólo en el punto 16 se encontró la fracción gasolina, en una concentración de 43.8 $\mathrm{mg} / \mathrm{kg}$. En cuanto a los HAP, sólo se encontró naftaleno $(0.99 \mathrm{mg} / \mathrm{kg})$ en el punto 16 , precisamente. No es de extrañar esta coincidencia, pues el naftaleno es un componente presente en las gasolinas mexicanas (Torres et al., 2002).

Tabla 1. Concentraciones de metales, H TP y otros hidrocarburos encontrados en el suelo de la TAD.

Todas en $\mathrm{mg} / \mathrm{kg}$. Excepto cuando se indica

\begin{tabular}{|c|c|c|c|c|c|c|c|c|}
\hline Punto & HTP & Diesel & Gasolina & Naftaleno & $\mathrm{Pb}$ & $\mathrm{Fe}$ & $\mathrm{Zn}$ & $\begin{array}{c}\text { Explosividad } \\
(\%)\end{array}$ \\
\hline 1 & 59,213 & ND & ND & ND & 10.45 & 6,262 & 26 & 100 \\
\hline 2 & 4,207 & 1,020 & ND & ND & 46.95 & 20,930 & 80.7 & 5 \\
\hline 3 & ND & $N D$ & ND & ND & 9.4 & 6,346 & 25.8 & 100 \\
\hline 4 & 10,003 & 2,443 & ND & ND & ND & 4,899 & 24.3 & 100 \\
\hline 5 & 29 & ND & ND & ND & ND & 6,162 & 21.4 & 5 \\
\hline 6 & ND & ND & ND & ND & 18 & 12,430 & 37.1 & 70 \\
\hline $6 b$ & ND & ND & ND & ND & 11 & 8,084 & 26.5 & ND \\
\hline 7 & ND & ND & ND & ND & 9.3 & 7,883 & 25 & ND \\
\hline 9 & ND & ND & ND & ND & 9.2 & 7,484 & 24 & ND \\
\hline 10 & 104 & ND & ND & ND & 8.3 & 6,504 & 20 & ND \\
\hline 11 & ND & ND & ND & ND & 10.8 & 10,010 & 33 & ND \\
\hline 12 & 33 & ND & ND & ND & 25 & 13,820 & 32 & ND \\
\hline 14 & ND & ND & ND & ND & ND & 4,885 & 18.5 & 30 \\
\hline 15 & 4,394 & 5,272 & ND & ND & 13.7 & 8,396 & 28 & ND \\
\hline 16 & 988 & ND & 43.8 & 0.99 & 37.8 & 12,680 & 40 & ND \\
\hline 17 & ND & ND & ND & ND & 15.6 & 8,825 & 29.7 & ND \\
\hline 18 & 64 & ND & ND & ND & 14.5 & 13,690 & 31.5 & ND \\
\hline 19 & ND & ND & ND & ND & 13.6 & 10,620 & 30.9 & ND \\
\hline 20 & ND & ND & ND & ND & ND & 6,396 & 21.7 & ND \\
\hline 21 & 48 & ND & ND & ND & ND & 5,277 & 21.3 & ND \\
\hline Min & ND & $N D$ & ND & ND & $N D$ & 4,885 & 18.5 & ND \\
\hline Max & 59,213 & 5,272 & 43.8 & 0.99 & 46.95 & 20,930 & 80.70 & 100 \\
\hline
\end{tabular}


DOI: http://dx.doi.org/10.22201/fi.25940732e.2006.07n1.001

Análisis de riesgo a la salud de una instalación petrolera del Suroeste de México

Tabla 1. Concentraciones de metales, H TP y otros hidrocarburos encontrados en el suelo de la TAD. Todas en $\mathrm{mg} / \mathrm{kg}$. Excepto cuando se indica (...continuación)

\begin{tabular}{ccccccccc}
\hline Punto & HTP & Diesel & Gasolina & Naftaleno & Pb & Fe & Zn & $\begin{array}{c}\text { Explosividad } \\
(\%)\end{array}$ \\
\hline Prom & 3,954 & 436.75 & 43.8 & 0.99 & 12.68 & 9,079 & 30.12 & 20.5 \\
$\begin{array}{c}\text { Desv } \\
\text { estándar }\end{array}$ & 13,241 & 1,277 & - & - & 12.29 & 4.002 & 13.32 & 38.0 \\
$\begin{array}{c}\text { Niveles } \\
\text { permitidos }\end{array}$ & 2,000 & - & - & 100 & 400 & 25,000 & 2,500 & - \\
\hline
\end{tabular}

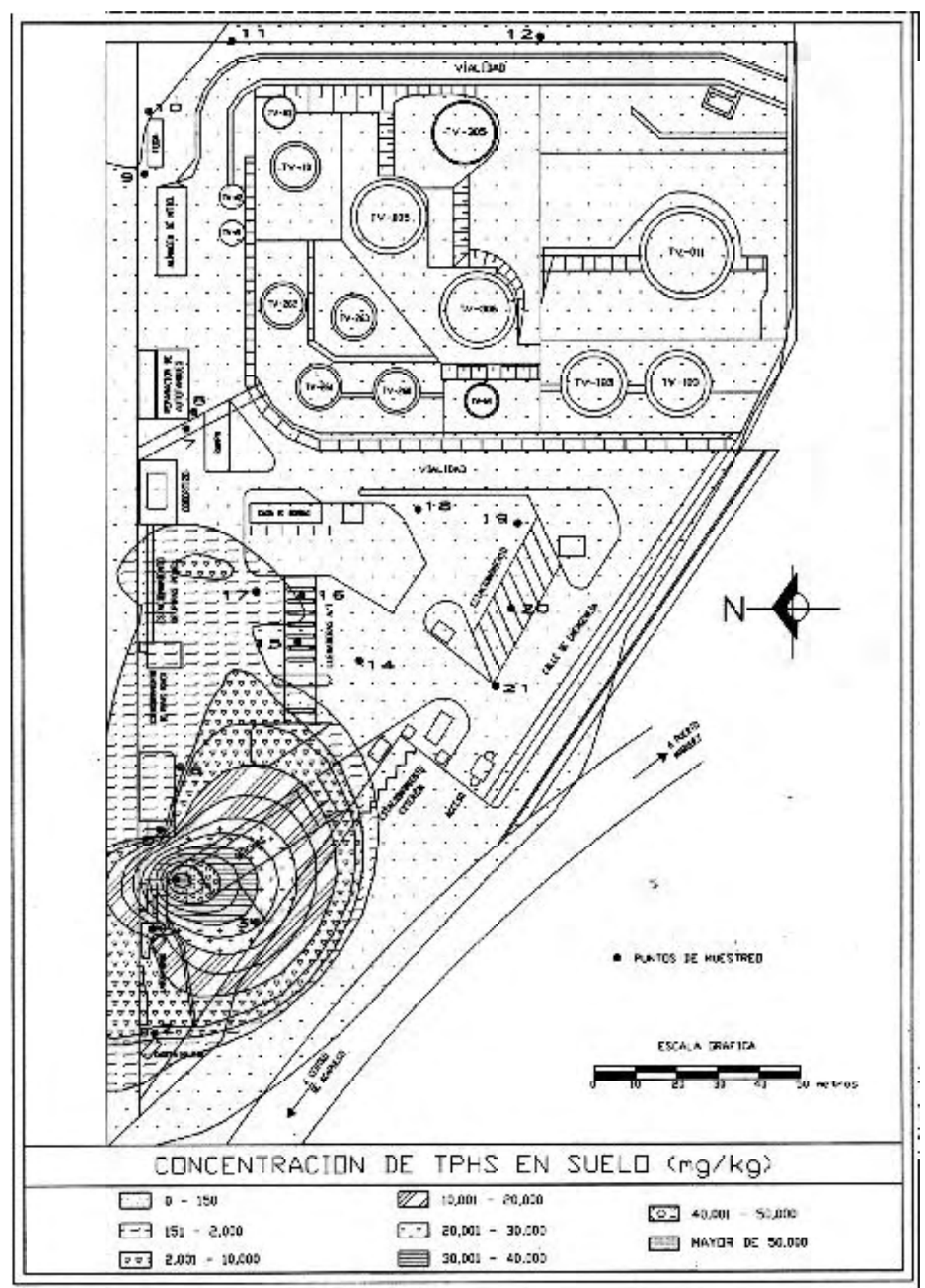

Figura 1. Distribución de HTP en la TAD 
Los Criterios Interinos para la Remediación de Suelos (CIRS), emitidos por la PFPA (1998), sugieren un valor de $2,000 \mathrm{mg} / \mathrm{kg}$, como HTP para suelos de tipo in dus trial, por lo que se puede decir que algunos puntos de la TAD se encuentran por encima de este valor. En cuanto al diesel y la gasolina, los mismos criterios establecen valores de 2,000 y $500 \mathrm{mg} / \mathrm{kg}$, respectivamente. Algunos valores de concentración de die sel en la TAD exceden esta recomendación.

Los valores de explosividad detectados en el suelo de la TAD se presentan en la tabla 1. Dichos valores estuvieron en el intervalo de ND a $100 \%$. Particularmente, los puntos 1,3 y 4 presentaron valores del $100 \%$, lo que coincide con los valores altos de HTP localizados en dos de los puntos (1 y 4). Los puntos 6 y 14 presentaron valores de explosividad de 30 y $60 \%$, respectivamente. Los puntos 2 y 5 presentaron valores del $5 \%$ y en el resto de los puntos no se detectó explosividad. Como puede verse, existe correlación entre la explosividad medida y el valor de HTP medidos en el suelo. Esta técnica permite hacer una estimación rápida de las zonas donde presumiblemente existe contaminación por hidrocarburos.

\section{Distribución de metales pesados}

En cuanto a los niveles de metales pesados encontrados en el suelo de la TAD se puede decir lo siguiente: No se encontró cromo en ninguna de las muestras analizadas. El Fe se encontró en concentraciones de 4,885 a 20,930 mg/kg, con un promedio de $9,079+/-4,000 \mathrm{mg} / \mathrm{kg}$. El Zn fue encontrado en concentraciones de entre 18.5 y 80.7 $\mathrm{mg} / \mathrm{kg}$ (con un promedio de $30.1+/-13.3 \mathrm{mg} / \mathrm{kg}$ ). Finalmente, el $\mathrm{Pb}$ se encontró en concentraciones de entre ND y $46.9 \mathrm{mg} / \mathrm{kg}$, con un promedio del $2.7+/-12.3 \mathrm{mg} / \mathrm{kg}$. En las figuras $2 \mathrm{a}, 2 \mathrm{~b}$ y $2 \mathrm{c}$ se presenta la distribución espacial de estos tres metales pesados en los suelos de la refinería. Los valores reportados en los CIRS (PFPA, 1998) para el $\mathrm{Zn}$ y Pb en suelos industriales son de 1,500 y $1,500 \mathrm{mg} / \mathrm{kg}$, respectivamente. Los valores límite reportados por Buonicore (1996) para Fe, Zn y Pb son de 25,000, 2,500 y $400 \mathrm{mg} / \mathrm{kg}$, respectivamente. Si se comparan los datos de la TAD con los valores para Fe, Zn y $\mathrm{Pb}$ reportados por Buonicore, todos los valores se encuentran dentro de los límites sugeridos.
Muller (1969), sugirió un parámetro para la evaluación de la contaminación por metales en suelos y sedimentos, llamado índice de geoacumulación (Igeo) y definido como:

$$
\text { lgeo }=\log _{2}(C n / 15 \times C b)
$$

Donde $\mathrm{Cn}$ es la concentración presente en el suelo o sedimento presumiblemente contaminado y $\mathrm{Cb}$ es el valor de la concentración de metal en el suelo basal (no contaminado). El factor de $1.5 \mathrm{da}$ un margen de cambio del $50 \%$.

De los datos de la antigua TAD, se seleccionaron aquellos puntos en donde no se reportaron HTP y este grupo se consideró como el suelo no contaminado. En la tabla 2 se presentan dichos puntos, los valores de concentración promedio y los mínimos y máximos encontrados.

Al aplicar los promedios de los valores de concentración encontrados en la TAD (Cn) y los promedios del suelo no contaminado (Cb) se calcularon los valores de Igeo para cada metal. Como puede observarse, los valores encontrados para el plomo, hierro y zinc fueron de -0.3492 , -0.5193 y -0.4894 , respectivamente. Estos valores con signo negativo indican no-acumulación.

Tabla 2. Contenido de metales pesados en suelos no contaminados

\begin{tabular}{cccc}
\hline Punto & $\mathrm{Pb}(\mathrm{mg} / \mathrm{kg})$ & $\mathrm{Fe}(\mathrm{mg} / \mathrm{kg})$ & $\mathrm{Zn}(\mathrm{mg} / \mathrm{kg})$ \\
\hline 3 & 9.4 & 6,346 & 25.8 \\
6 & 18.0 & 12,430 & 37.1 \\
$6 \mathrm{~b}$ & 11.0 & 8,084 & 26.5 \\
7 & 9.3 & 7,883 & 25.0 \\
9 & 9.2 & 7,484 & 24.0 \\
11 & 10.8 & 10,010 & 33.0 \\
17 & 15.6 & 8,825 & 29.7 \\
19 & 136 & 10,620 & 30.9 \\
M in & 9.2 & 6,346 & 24.0 \\
Max & 18.0 & 12,430 & 37.1 \\
Promedio & 12.11 & 8,960 & 29.0 \\
Desv est & 3.29 & 1,958 & 4.52 \\
Igeo & -0.3494 & -0.5193 & -0.4898 \\
\hline
\end{tabular}


Si se comparan los valores de $\mathrm{Fe}, \mathrm{Pb}$ y $\mathrm{Zn}$ con los valores de HTP para todos los puntos evaluados, no se encuentra una buena correlación $(r 2=0.0774,0.04336$ y 0.0092 , respectivamente), lo que sugiere que el origen de los metales no es la contaminación por hidrocarburos. Este hecho apoya lo anteriormente discutido, con respecto al índice de geoacumulación. Por otro lado, los valores de $\mathrm{Fe} \mathrm{y} \mathrm{Pb}$, y los de $\mathrm{Fe}$ y $\mathrm{Zn}$ están fuertemente relacionados entre sí $\left(\mathrm{r}^{2}\right.$ $=0.8133$ y 0.8352 , respectivamente), lo que puede indicar que los tres metales tienen el mismo origen. Con respecto a las figuras $2 \mathrm{a}, 2 \mathrm{~b}$ y $2 c$, se observa que las distribuciones de los metales pesados no son iguales, pero guardan cierta correspondencia. Esto haría pensar que se trata de los valores de concentración naturales del suelo.

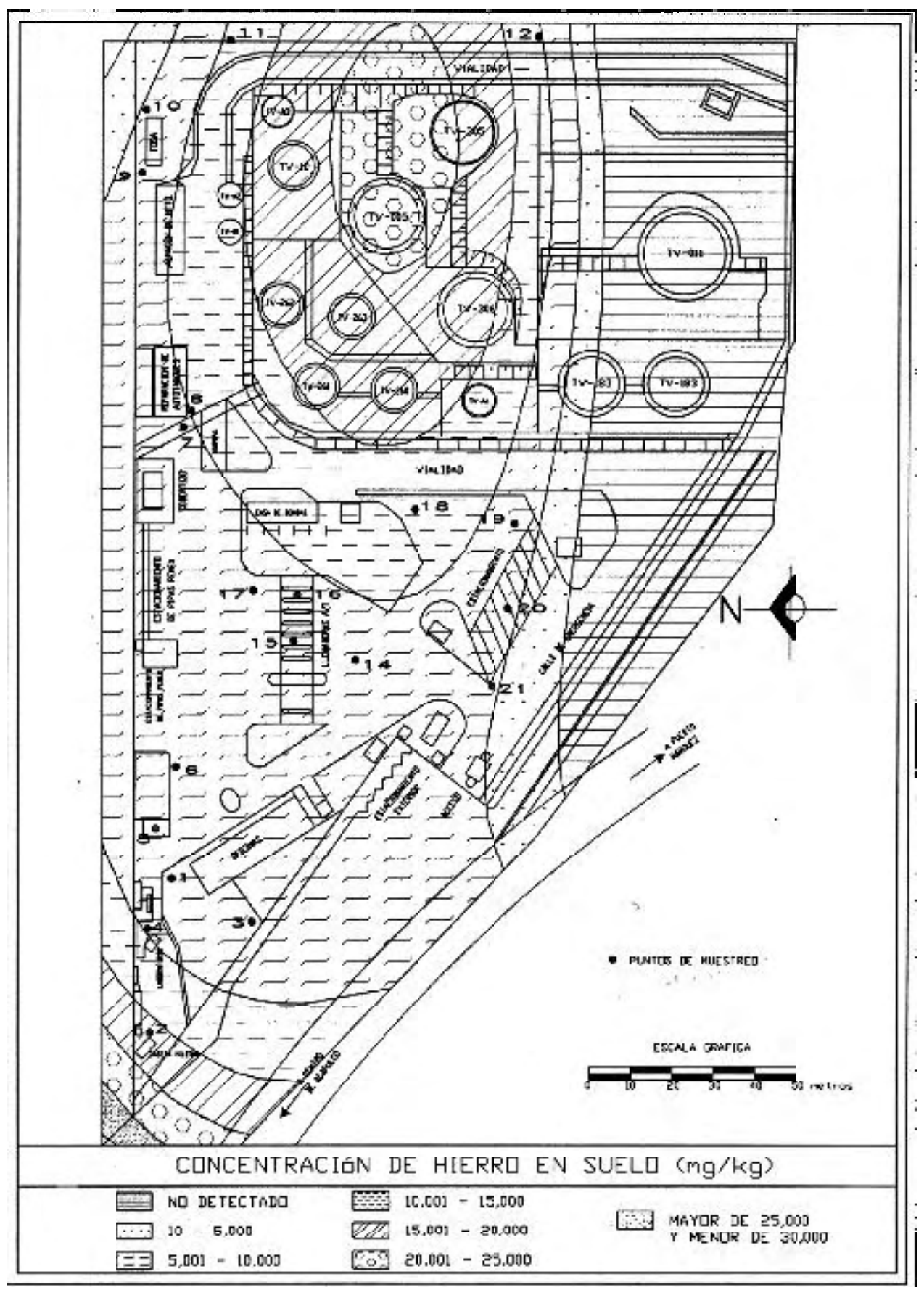

Figura 2a. Distribución de hierro en la antigua TAD 


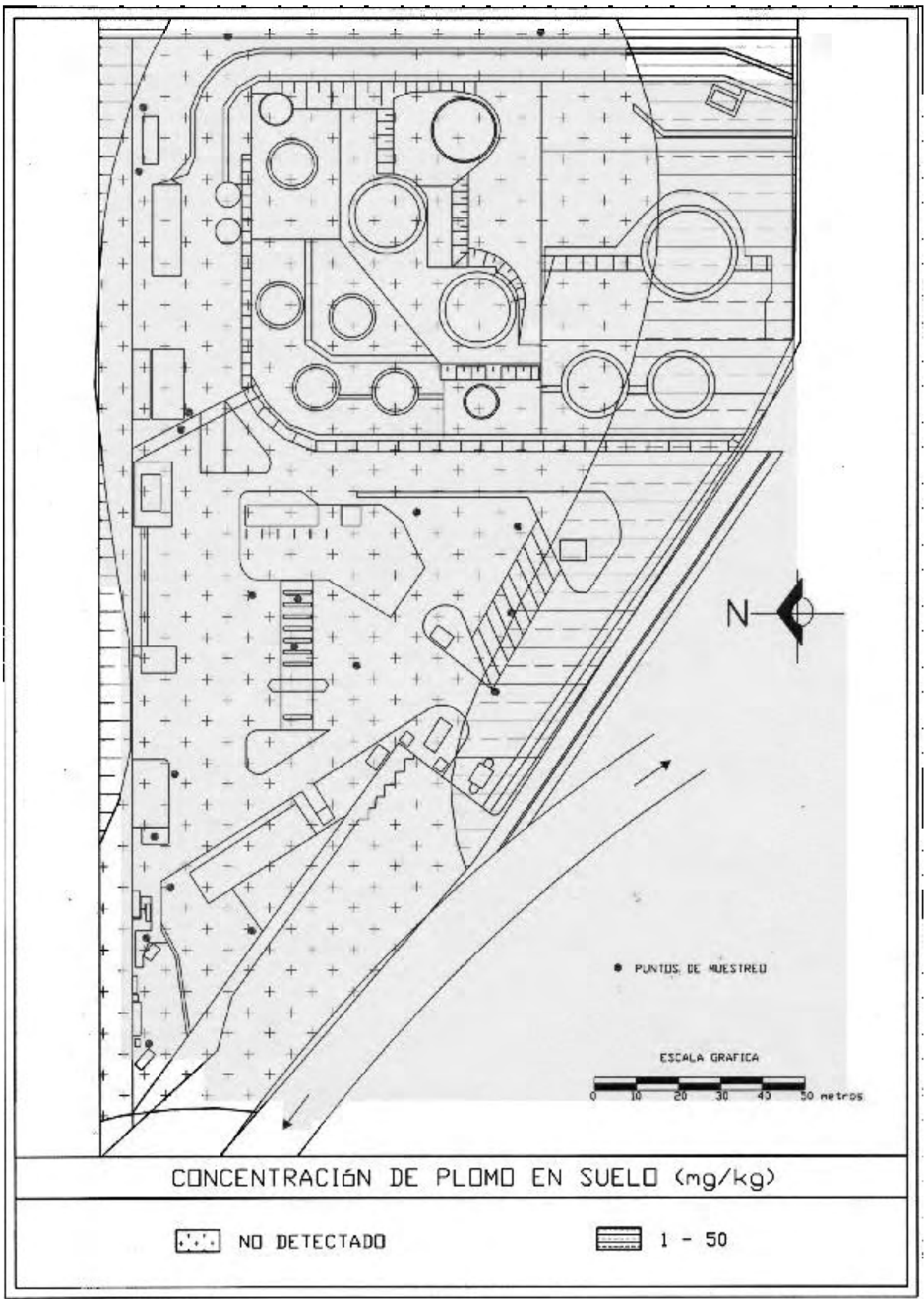

Figura 2b. Distribución de plomo en la antigua TAD 


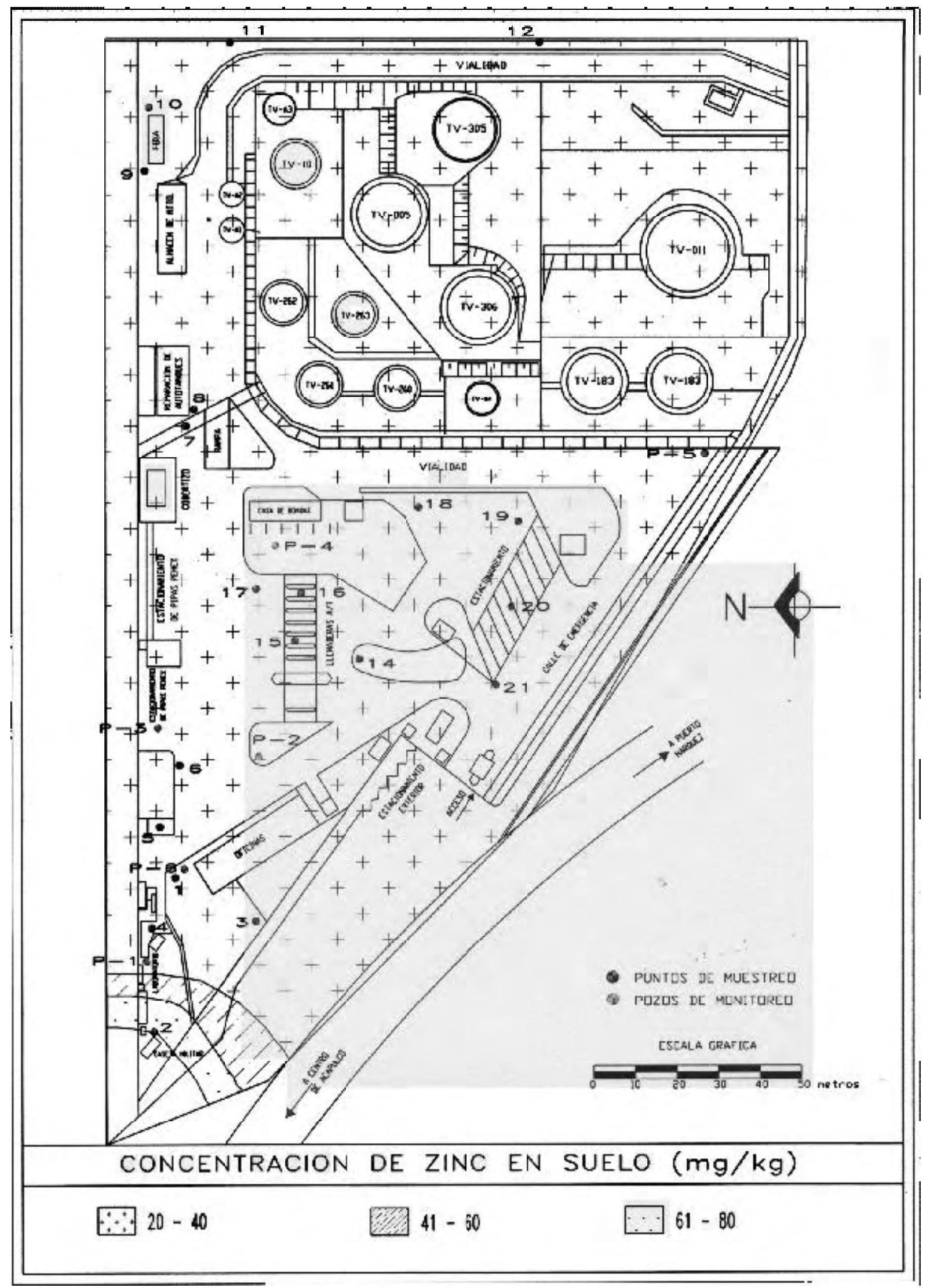

Figura 2c. Distribución de zinc en la antigua TAD 


\section{Agua subterránea}

En la tabla 3 se presentan las concentraciones de HTP, Fe y Zn en los 6 pozos distribuidos en la TAD. También se presenta el nivel de agua encontrado en cada pozo y su valor de pH (medición de campo). En el punto 3, casi no había agua, pero sí fue posible tomar muestra para realizar los análisis correspondientes. Como se observa, el promedio de nivel en los pozos fue de $2.7+/-1.4 \mathrm{~m}$, aunque el pozo 5 presentó una profundidad de $4.3 \mathrm{~m}$. El intervalo de pH medido en campo es de 5.9 a 6.5, por lo que se considera a las aguas subterráneas como ligeramente ácidas. Con respecto a los HTP, el intervalo va de $0.3 \mathrm{mg} / \mathrm{l}$ (punto 4) a $21.2 \mathrm{mg} / \mathrm{l}$ (punto 5). El promedio observado fue de $5.3+/-8.4$ $\mathrm{mg} / \mathrm{l}$. De acuerdo con Buonicore (1996), la concentración máxima permisible de HTP en aguas subterráneas varía entre 1 y $10 \mathrm{mg} / \mathrm{l}$, por lo que se puede decir que las aguas subterráneas de la TAD se encuentran dentro del intervalo permitido. No se detectó la presencia de $\mathrm{Cr}$ en las aguas subterráneas, pero sí de Fe y Zn. El hierro fue encontrado en concentraciones de 0.19 a $6.37 \mathrm{mg} / \mathrm{l}$ (con un promedio de $3.2+/-3.4 \mathrm{mg} / \mathrm{l}$ ), mientras que el zinc, en un intervalo de 0.03 a 0.11 (con un promedio de $0.07+/-0.04 \mathrm{mg} / \mathrm{l}$ ).

Con el fin de encontrar relaciones existentes entre los parámetros antes descritos, se hicieron correlaciones lineales entre ellos y se calcularon los valores de $r^{2}$. En cuanto al $\mathrm{pH}$ de las aguas subterráneas, se encontró que éste no se relaciona con el nivel de metales encontrados (Fe y Zn), pero sí con el contenido de HTP $\left(r^{2}=0.9176\right)$. Este hecho sugeriría que entre más ácidas son las aguas subterráneas son capaces de disolver mayor cantidad de compuestos presentes en los hidrocarburos depositados en los suelos. Por otro lado, los valores de Fe y Zn no correlacionaron con el contenido de HTP en las aguas $\left(r^{2}=0.1452\right.$ y 0.1984 , para $\mathrm{Fe}$ y $\mathrm{Zn}$, respectivamente), lo que puede indicar que el origen de los metales no está relacionado con los derrames de hidrocarburos en el suelo de la antigua TAD. Este hecho confirma lo encontrado al tratar de correlacionar el contenido de metales y el valor de HTP presentes en el suelo. Es interesante agregar, que el valor de Fe y $\mathrm{Zn}$ en las aguas, tampoco correlacionó $\left(r^{2}=0.4058\right)$, lo que indicaría que los dos metales provienen de diferentes fuentes. Por último, se encontró que el nivel de agua encontrado en los pozos no correlacionó con el pH, los TPHs, ni el contenido de $\mathrm{Zn}$ de las aguas $\left(\mathrm{r}^{2}=0.3843,0.1874 \mathrm{y}\right.$ 0.680 , respectivamente), pero sí con el contenido del $\mathrm{Fe}\left(\mathrm{r}^{2}=0.8997\right)$, lo que sugeriría que el origen del hierro en el agua es el lavado del propio subsuelo de la antigua TAD.

\section{Análisis de riesgo a la salud}

En la tabla 4, se muestran los riesgos correspondientes por contaminante y por ruta de

Tabla 3. Niveles de contaminación del agua en la TAD

\begin{tabular}{ccccccccc}
\hline Pozo & $\begin{array}{c}\text { Nivel de } \\
\text { agua }\end{array}$ & $\mathrm{pH}$ & $\begin{array}{c}\mathrm{HTP} \\
(\mathrm{mg} / 1)\end{array}$ & $\begin{array}{c}\mathrm{Pb} \\
(\mathrm{mg} / 1)\end{array}$ & $\begin{array}{c}\mathrm{Fe} \\
(\mathrm{mg} / 1)\end{array}$ & $\begin{array}{c}\mathrm{Cr} \\
(\mathrm{mg} / 1)\end{array}$ & $\begin{array}{c}\text { Zn } \\
(\mathrm{mg} / 1)\end{array}$ & $\begin{array}{c}\text { Explosividad } \\
\text { vapores } \\
(\%)\end{array}$ \\
\hline 1 & 2.5 & 6.4 & 0.64 & $\mathrm{ND}$ & 6.37 & $\mathrm{ND}$ & 0006 & $\mathrm{ND}$ \\
2 & 3.1 & 6.6 & 0.84 & $\mathrm{ND}$ & 2.29 & $\mathrm{ND}$ & 0.044 & $\mathrm{ND}$ \\
3 & 0 & 6.4 & 8.35 & $\mathrm{ND}$ & 8.4 & $\mathrm{ND}$ & 0.112 & $\mathrm{ND}$ \\
4 & 3.3 & 6.4 & 0.3 & $\mathrm{ND}$ & 0.39 & $\mathrm{ND}$ & 0.113 & $\mathrm{ND}$ \\
5 & 4.3 & 5.9 & 21.2 & $\mathrm{ND}$ & 0.19 & $\mathrm{ND}$ & 0.035 & $\mathrm{ND}$ \\
6 & 3.1 & 6.5 & 0.49 & $\mathrm{ND}$ & 1.54 & $\mathrm{ND}$ & 0.034 & $\mathrm{ND}$ \\
$\begin{array}{c}\text { Promedio } \\
\text { Desv }\end{array}$ & 2.72 & 6.37 & 5.3 & - & 3.2 & - & 0.07 & - \\
estándar & 1.45 & 0.24 & 8.4 & - & 3.4 & - & 0.04 & - \\
$\begin{array}{c}\text { Nivel } \\
\text { Permisible }\end{array}$ & - & & $1-10$ & 0.025 & 0.30 & 0.05 & 5.0 & - \\
\hline
\end{tabular}


exposición, así como los niveles de limpieza basados en riesgo para el suelo y agua subterránea. Los resultados sombreados indican que se ha excedido el riesgo aceptable y la concentración que asegura este riesgo en el medio afectado. Se puede observar que, de acuerdo con el mo delo RBCA Tool Kit for Chem i cal Re leases, las concentraciones de naftaleno y zinc presentes en el suelo y agua subterránea, no representan un riesgo para la salud de los habitantes de las colonias aledañas ni para los trabajadores de la TAD (no se rebasó el límite establecido como aceptable de 1.0).

\section{Áreas y volúmenes a tratar}

A partir de los análisis realizados en las muestras de suelo, se calcularon las áreas y volúmenes a tratar en la TAD por medio del programa Rock Works. Si se consideran las áreas contaminadas por HTP en concentraciones mayores o iguales a $2,000 \mathrm{mg} / \mathrm{kg}$, que es el límite propuesto por los CIRS, se estima que es necesario sanear un área total de $3,289 \mathrm{~m}^{2}$, que considerando una profundidad de $2.48 \mathrm{~m}$, dan como volumen de suelo a tratar, la cifra de 8,157 $\mathrm{m}^{3}$.

\section{Técnicas de rehabilitación propuestas}

Ya que la mayor parte de los suelos contaminados con más de $2,000 \mathrm{mg} / \mathrm{kg}$ se encuentra en la zona de estacionamientos exteriores, estacionamientos de pipas y zonas de llenaderas, se pueden utilizar técnicas de saneamiento ex situ e in situ.

Una técnica que tiene gran potencial de aplicación en casos como el de la TAD es el proceso de biopilas. Fundamentalmente se trata de la intensificación del proceso natural de biodegradación, que se da cuando los microrganismos autóctonos del suelo entran en contacto con los contaminantes presentes en el suelo. Para intensificar este proceso es necesario examinar aspectos tales como la adecuada relación de los principales macronutrientes (CNP), la humedad, la temperatura y la presencia de oxígeno. Una serie de análisis y experiencias a nivel de laboratorio, permiten determinar las condiciones óptimas para que el proceso de biodegradación se lleve a cabo. Para mas información del proceso de biopilas, referirse a Iturbe et al. (2002).

En cuanto a las aguas subterráneas, en 5 de los 6 pozos de monitoreo se rebasa el nivel máximo permisible de Fe propuesto para el agua potable (NOM-127-SSA1-94), que es de $0.3 \mathrm{mg} / \mathrm{l}$, por lo que se requerirá reducir la concentración de hierro en el agua subterránea. Para realizar esta disminución, se han propuesto varios métodos fisicoquímicos, como la adsorción en columnas empacadas con resinas sintéticas, o bien, con óxidos metálicos que retienen al hierro, o por precipitación. Es importante determinar si el origen del Fe en las aguas es el suelo de la zona, revisando los contenidos de este metal en aguas subterráneas de otros sitios cercanos y lejanos a la antigua TAD.

\section{Conclusiones}

El suelo de la TAD se encuentra contaminado por HTP en concentraciones de ND a 59,000 mg/kg. La zona más contaminada coincide con el punto 1 , localizado en la zona verde, al Norte de las oficinas. Los puntos 2 y 4 , junto a la planta de tratamiento de efluentes también están altamente

Tabla 4. Riesgo y concentraciones de limpieza a alcanzar en Ia TAD

\begin{tabular}{|c|c|c|c|c|c|c|c|c|c|c|c|}
\hline \multirow[t]{4}{*}{ Compuesto } & \multirow[t]{4}{*}{ Clase } & \multicolumn{8}{|c|}{ Riesgo I índice de peligrosidad (HI) } & \\
\hline & & \multirow{3}{*}{$\begin{array}{c}\text { HQ } \\
\text { Riesgo }\end{array}$} & \multicolumn{2}{|c|}{ Agua Subterránea } & \multirow{2}{*}{\multicolumn{2}{|c|}{$\begin{array}{c}\text { Suelo } \\
\text { On-site } \\
(0 \mathrm{~m}) \\
\end{array}$}} & \multicolumn{3}{|c|}{ Aire exterior } & & \\
\hline & & & $\begin{array}{c}\text { On-site } \\
(0 \mathrm{~m})\end{array}$ & $\begin{array}{l}\text { Off-site } 1 \\
(84 \mathrm{~m})\end{array}$ & & & & $\begin{array}{l}\text { n-site } \\
(0 \mathrm{~m})\end{array}$ & $\begin{array}{l}\text { O ff-site } \\
(84 \mathrm{~m})\end{array}$ & $\begin{array}{l}\text { con base en } \\
\text { Agua }\end{array}$ & $\begin{array}{l}7 \text { el riesgo } \\
\text { Suelo }\end{array}$ \\
\hline & & & Comercial & Residencial & Comercial & $\begin{array}{l}\text { Trabajadores } \\
\text { construcción }\end{array}$ & Comercial & $\begin{array}{l}\text { Trabajadores } \\
\text { construcción }\end{array}$ & Comercial & & \\
\hline Naftaleno & D & HQ & $4.4 \times 10-4$ & $5.5 \times 10_{-4}$ & $9.1 \times 10^{-6}$ & $7.4 \times 10^{-6}$ & $9.7 \times 10_{-5}$ & $6.0 \times 10^{-5}$ & $1.1 \times 10_{-4}$ & $>3.0 \times 10_{-1}$ & $1.5 \times 10-4$ \\
\hline Zinc & D & $\mathrm{HQ}$ & $1.2 \times 10^{-2}$ & $1.5 \times 1^{-2}$ & $1.3 \times 10^{-4}$ & $1.9 \times 10^{-4}$ & - & - & & $2.4 \times 10^{-1}$ & $5.3 \times 10^{-3}$ \\
\hline Total & & & $1.3 \times 10^{-2}$ & $1.6 \times 10^{-2}$ & $1.4 \times 10^{-4}$ & $2.0 \times 10^{-4}$ & $9.7 \times 10^{-5}$ & $6.0 \times 10^{-5}$ & $1.1 \times 10^{-4}$ & & \\
\hline
\end{tabular}


contaminados. No se detectó la presencia de BTEX, MTBE ni MTE. De los 16 HAP s analizados, sólo se encontró la presencia de naftaleno en el punto 16, a una concentración por debajo de $1 \mathrm{mg} / \mathrm{kg}$.

Se identificó la presencia de $\mathrm{Pb}$, Fe y $\mathrm{Zn}$ en el suelo, pero los índices de geo-acumulación calculados $(-0.3492,-0.5193$ y -0.4894 , respectivamente), sugieren que se trata de concentraciones naturales del suelo y no de contaminación alguna. Se encontraron HTP en las aguas subterráneas, solo en uno de los 6 puntos por arriba del nivel recomendado de $10 \mathrm{mg} / \mathrm{l}$. También se encontró que el Fe excede el valor sugerido de 0.3 $\mathrm{mg} / \mathrm{l}$ en 5 de los 6 pozos estudiados. Se encontró que el pH de las aguas subterráneas no está relacionado con el nivel de metales encontrados (Fe y $\mathrm{Zn})$, pero sí con el contenido de HTP $\left(\mathrm{r}^{2}=\right.$ 0.9176). Si se consideran las áreas contaminadas por HTP en concentraciones mayores 0 iguales a $2,000 \mathrm{mg} / \mathrm{kg}$, que es el límite propuesto por los CIRS, se estima que es necesario sanear un área total de $3,289 \mathrm{~m}^{2}$, que considerando una profundidad de $2.48 \mathrm{~m}$, dan como volumen de suelo a tratar la cifra de $8,157 \mathrm{~m}^{3}$.

El análisis de riesgo a la salud indicó que las concentraciones de naftaleno y zinc, presentes en el suelo y agua subterránea, no representan un riesgo para la salud de los habitantes de las colonias aledañas ni para los trabajadores de la TAD. La técnica biopila se recomienda como opciones adecuadas para el saneamiento del suelo contaminado. En cuanto al contenido de hierro en las aguas subterráneas, habría que utilizar un método de columnas de intercambio, o bien, la precipitación del metal para su remoción.

\section{Agradecimientos}

Agradecemos ampliamente la participación de D. Rodríguez (II/NAM) en la elaboración de planos y revisión del texto.

\section{Referencias}

Adams R.H., Domínguez V.I. y García L. (1999). Potencial de la biorremediación de suelo y agua impactados por petróleo en el trópico mexicano. Terra 17(2), pp. 159-174.
Adenyi A.A. and Afolabi J.A. (2202). Determination of Total Petroleum Hydrocarbons and Heavy Metals in Soils Within the Vicinity of Facilities Handling Refined Petroleum Products in Lagos Metropolis. Environ mentInternational, 28, pp. 79-82.

ASTM American Society of Testing and Mate rials (1996). Standard Guide for Risk Based Corrective Action Applied at Petroleum Release Sites. E1739-95, Penn sylvania, USA.

Bakker M.I., Casado B., Koerselman J.W., Tolls J. and Kollofel C. (2000). Polycyclic Aromatic Hydro car bons in Soil and Plant Samples From the Vecinity of an Oil Refinery. The Science of the Total Environment, 263, pp. 91-100.

Buonicore A.J. (1996). (Editor) Cleanup Criteria for Contaminated Soil and Groundwater. ASTM,Pensilvania, EUA.

Crane J.L. and Tuchman M. (1993). Human Health Risk Assessment: Bufalo River, New York, Area of Concern. EPA- 905-R93-008. Seattle, Washington, Internet: http://www. Epa.gov/glnpo/arcs/EPA-905-R93-008/EPA -905-R93-008.html

Chen Z., Huang G.H. and Chakma A. (2000). Risk Assessment of a Petroleum-Contaminated Site Through a Multi-Phase and MultiComponent Modelling Approach. Journal of Petro leum S cienceand E ngineering, 26, pp. 273-281.

Ekundayo E.O. and Obuekwe C.O. (1997). Effects of an Oil Spill on Soil Physico- Chemical Properties of a Spill Site in a Typic Paleudult of Midwestern Nigeria. Environmental M oni toring and A ssess ment, 45, pp. 209-221.

Gutiérrez M.C. y Zavala J. (2001). Rasgos hidromórficos de suelos tropicales contaminados con hidrocarburos. Terra 20, pp. 101-111.

GSI Ground Water Services Inc. (1998). RBCA Tool Kit for $C$ hem ical Rel eases $G$ uid ance M anual. Ground Water Services Inc, United States of America.

Iturbe M.R., Flores R.M., Flores C.R., Ro- dríguez R.D., Chávez C., Rocha M. y Pérez G. (1999). Evaluación de la contaminación en el suelo y subsuelo de la terminal de almacenamiento y distribución de Puebla, Puebla. PEMEXRefinación, Informe Con-fidencial.

Iturbe R., Flores R.M. and Torres L.G. (2003a). Soil and Water Contam ination Levels in an Out-of-Service 
Oil Distribution and Storage Station in Michoacan, Mexico. Water, Air and Soil Contamination. 146(1-4), pp. 261.281.

Iturbe R. , Flores R.M. and Torres L.G. (2003b). Subsoil Contaminated by Hydrocarbons in an Out-of Service Oil Distribution and Storage Station in Zacatecas, México. Environmental M onitoring and Assessment .44, pp. 608-620.

Iturbe R, Flores C., Chavez C., y Roldan A. (2002). Saneamiento de suelos contaminados con hidrocarburos mediante biopilas. Ingeniería Investigación $y$ Tecnología, Vol. III, No. 1, Ene-Mar.

Lesser J.M. y Saval S. (2000). Caracterización y comportamiento de la contaminación con hidrocarburos en un suelo arcilloso. Ingeniería y Ciencias A mbientales. 51, pp. 25-29.

Muller G. (1969). Index of Geoaccumulation in Sediments of the Rhine River. Geojournal, 2, pp. 108-118.

NRC (1983). National Research Council. Risk Assessment in the Federal Goverment: M anaging the Process. National Academy Press. Washington D.C., USA.

PEMEX (2001). Informe de actividades 2001. Internet: htpp//www.PEMEX.com.mx

PFPA (1998). Procuraduría Federal de Protección al Ambiente. Restauración de Suelos Contaminados. Internet: http:/www. profepa.gob, mx/saa/audita43.htm

Sharma V.K., Hicks S.D., Rivera W. and Vazquez F.G. (2002). Characterization of Petroleum Hydrocarbons Following an Oil Spill into a Coastal Environment of South Texas, USA. Water, A ir and S oil Pollu tion, 134, pp. 111-127.

Shriadah M.A. (1999). Oil Contamination Along Oil Tanker Routes of the United Arab Emirates (The Arabian Gulf and the Gulf of Oman). Bulletin of Environmental Contamination and Toxicollogy, 63, pp. 203-210.

Torres L.G., Castro B., Pérez S. and Iturbe R. (2002). Characterization of Maya crude and five Mexican Oil Frac tions for Envi ron mental Forensic Applica tions. En preparación. 
DOI: http://dx.doi.org/10.22201/fi.25940732e.2006.07n1.001

R. Iturbe-Argüelles, R.Ma. Flores-Serrano y L.G. Torres-Bustillos

\section{Semblanza de los autores}

Rosario Iturbe-Argüelles. Ingeniera civil y doctora en ingeniería egresada de la Facultad de Ingeniería, UNAM es investigado ra del área de ingeniería ambiental en el Instituto de Ingeniería, principalmente dedicada a la caracterización y saneamiento de suelos contaminados por hidrocarburos desde hace más de diez años. Actualmente dirige el grupo de saneamiento de suelos y acuíferos en el que participan más de 20 académicos y alumnos de posgrado.

Rosa Maria Flores-Serrano. Es ingeniera indus trial en producción por el Instituto Tecnológico de Mérida, desde 1991. En 1996, obtuvo con honores la maestría en la División de Estudios de Posgrado de la Facultad de Ingeniería de la UNAM. En el año 2005, se graduó como doctora en ingeniería (Ambiental) dentro del Programa de Maestría y Doctorado en Ingeniería de la UNAM. Actualmente colabora en el grupo de trabajo saneamiento de suelos y acuíferos de la Coordinación de Ingeniería Ambiental del Instituto de Ingeniería, UNAM, participando en proyectos relacionados con el comportamiento de metales e hidrocarburos en el suelo y agua subterránea. Ha participado como instructora en el tema de evaluaciones de riesgo en diversos cursos, entre los cuales se encuentran los Cursos Regionales Sobre Manejo Adecuado de Residuos, organizados por INE-SEMARNAT y JICA. Desde el año 2002, es miembro del Grupo Técnico de integración del proyecto de norma oficial mexicana, el cual establece criterios para determinar las concentraciones de remediación de suelos contaminados por diversos metales y metaloides. Es miembro de la Asociación Mesoamericana de Ecotoxicología y Química Ambiental (AMEQA), de la So ciety of Envi ron mental Toxi cology and Chem istry (SETAC), y de la Asso ci a tion for Envi ron mental Health and Sciences (AEHS).

Luis G ilberto Torres-Bustillos. Es licenciado en ingeniería química por la Benemérita Universidad Autónoma de Puebla, obtuvo el grado de maestría en biotecnología por la UACPyP/CCH, UNAM y el doctorado en ingeniería por la DEPFI/UNAM. Actualmente es investigador en la Coordinación de Ingeniería Ambiental del Instituto de Ingeniería, UNAM. Tiene experiencia en procesos fermentativos, reología y mezclado de medios complejos, tratamientos biológicos para aguas residuales industriales y caracterización/tratamiento de suelos contaminados con metales y/o hidrocarburos del petróleo. Ahora, se ha orientado a la aplicación de surfactantes a los problemas ambientales. Sus principales líneas de investigación son la Biodegradación de fracciones de petróleo en suelos atemperados, asistida por surfactantes, Lavado de suelos in situ y ex situ asistido con surfactantes, Preparación de emulsiones de fracciones de petróleo-surfactantes-agua, como primer paso para el biotratamiento de combus ti bles (i.e. desulfuración biológica) y Reología y mezclado de lodos y suspensiones, aplicados a problemas ambientales. 